\title{
Modeling and simulation of metal organic halide vapor phase epitaxy (MOHVPE) growth chamber
}

\author{
N. Zieyana Mohamed Annuar • \\ Mohd Faizul Mohd Sabri $\cdot$ A. Shuhaimi Abu Bakar
}

\begin{abstract}
Over the last few decades, there was a substantial appeal on the growth of gallium-nitride $(\mathrm{Ga}-\mathrm{N})$ based alloy for high performance optoelectronic devices such as blue/violet laser diode, blue/white light emitting diode etc. In the recent years, there have been revolutionary changes in semiconductor field. Growth method for GaN-based film has been extensively explored, with success of thick film growth using halide vapor-phase epitaxy technique. The theoretical changes were attributed from the experimental results where modeling was vastly used for the purpose of design of equipment. This is because of the cost of the equipment and it is one of the major burdens in semiconductor processing. This process constitutes an important technology for manufacturing thin solid film in the semiconductor industry. To address these issues, a new development called metal organic halide vapor phase epitaxy (MOHVPE) reactor has been proposed in this study. Modeling with five inlet nozzles with $54 \mathrm{~cm}$ long is designed by design software. The numerical study of horizontal $\mathrm{MOH}-$ VPE growth shows dependence on temperature and species flow rates. The inlet area is set to room temperature while the whole chamber is set in the temperature range from 1,273 to $1,473 \mathrm{~K}$. Growth process reactor geometry that involved with temperature distribution stabilization and uniformity control flow pattern between the substrate holder are discussed. It is seen that the flow pattern
\end{abstract}

N. Zieyana Mohamed Annuar ( $₫)$. M. F. M. Sabri Department of Mechanical Engineering, Faculty of Engineering, University of Malaya, 50603 Kuala Lumpur, Malaysia e-mail:nzieanaz@gmail.com

A. Shuhaimi Abu Bakar

Department of Physics, Faculty of Science, Low Dimensional Research Centre, University of Malaya, 50603 Kuala Lumpur, Malaysia is influenced more by the temperature distribution and geometry of the chamber. The numerical study of horizontal MOHVPE growth shows a function of temperature and species flow rates has been performed with specific condition to find the ideal position of the substrate for growth process in future.

\section{Introduction}

Nowadays, semiconductor industry move step ahead in development thin films (Narayan and Wang 2002). It developed the layers on the wafer to produce a semiconductor component. Metal Organic Chemical vapor deposition (MOCVD) of a thin single-crystal silicon film is growth on single-crystal silicon substrate of the same crystallographic orientation. While further perfected to growth thick layer of wafer is perform in halide vapor-phase epitaxy (HVPE) method. In both research and industrial field, Metal Organic Vapor Phase Epitaxy (MOVPE) has developed into a viable and necessary technique for production of semiconductor device layers. The epitaxial structure is the heart of the semiconductor device layer and MOVPE is considered the enabling technology for epitaxial growth of compound semiconductor devices.

Based on previous research, the insufficiency of this method which is MOVPE and HVPE are identified. Lack of uniformity wafer curvature produced caused by lattice mismatch that came from MOVPE method (Taiyo et al. 1998). Thermal expansion will affect the maximum crack occurs and nevertheless the threading dislocation will appear in minimum stage inside growth chamber (Taiyo et al. 1998). These happen when $\mathrm{GaN}$ was reacting with sapphire as substrate. Further improvement is carried out in HVPE method by using $\mathrm{GaN}$ to replace sapphire as substrate, after 
thin film has grown on the $\mathrm{GaN}$ as substrate, the process is continued by cutting the wafer into slice. This processed have to go through up to four instruments to get the results with refinement wafer or thin film. Later on, process of chemical mechanical polishing (CMP) to obtain uniformity is done. The final process is MOVPE growth to grow layers (combination of layers) for devices, such as light emitting diode (LED), laser diode (LD) and high electron mobility transistor (HEMT) proceed to get the surface uniformity of wafer in future experiments (Technology of Gallium Nitride 2005)

In conjunction with reactor design geometry and several identified inefficiency by experimental process, several studies have been focusing on developing the simulation tools aimed towards the optimizing flow pattern to produce uniformity. Currently, the design modelling for process method only involved in every single reactor has been brought forward which are MOVPE and HVPE methods of growth reactor. To address these issues, new development called metal organic halide vapour phase epitaxy $(\mathrm{MOH}-$ VPE) has been proposed. These are the hybridization between the growth reactor for MOVPE as well as HVPE concurrently in a single reactor.

The evolution of new design software was accustomed to design and simulate the process run by numerical analysis. Attention is paid to the growth of the chamber itself for analysing the reliability to withstand the heating process based on the simulation run. Both the Modelling as well as the simulation methodologies are discussed for such type of complex structure. Some significant issues i.e. controlling of the simulation and the material model are addressed. The applications for MOHVPE are illustrated in the presented analytical software for virtual product exploitation projects by software simulations.

The advantage of such system is the possibility to simultaneously growthick (high growth rate) and thin (low growth rate) GaN-based layer on a target substrate, without exchanging growth instruments when both thick and thin layer is necessary on the same substrate. Therefore, it reduces surface contamination, layer stress and substratebowing during cool-down and heat-up process, resulting samples with a lower threading dislocation density and higher film quality. While in having thin and thick film growth mechanisms coupled into a single growth reactor significantly simplifies growth preparation, during interreactor substrate transfer/steps and significant cost-reduction due to the procurement of less instrument/parts and with lesser maintenance. Researchers are mostly focusing on wafer growth uniformity without considering the effect of structure and geometry analysis flow pattern for mechanical analysis side (Utsumi et al. 2004).

This research opens the future on creative innovation and exploration in metal-organic and hydride gaseous supply and control, thin and thick film growth techniques, electronic (HEMT) and optoelectronic (LED/LD/PD) devices fabrication in semiconductor research future ahead. Deposition technology is very useful and essential application in the electronic device manufacturing in the industries, especially to fabricate the semiconductor devices which are completely dependent on it. Thin solid films are formed from the deposition of various materials' gaseous, vapour, liquid and solidus phases in the semiconductor device manufacturing industries (Meyyappan 2000). In general, the gaseous phases of the materials contribute to grow the Epitaxial films of semiconductors. These films develop the layers on the wafer to produce a semiconductor component. Industrial and technological revolution has been observed in last few years. Semiconductor devices are also following that evolution and even helping other technical advancements to evolve further. The theoretical enrichment of the semiconductor devices are contributed from both the experimental setups as well as from modelling of the equipment design analysis significantly. Modelling, analysis and development of new devices in the semiconductor industries are encouraged due to increased financial burden of expenditures related to the sophisticated and advanced experimental set up establishment. The evolution of new equipment can often be achieved through trial and error procedure from the experimental set up utilization which is both time consuming as well as expensive, too. But this equipment design can be process optimized, efficient, less time consuming and less experimental work demanding once a reliable modelling is built. The models are accepted after effective validation process and then the model can be utilized for more parametric analysis to establish knowledge based technology for the process variables against their performances. For further observation on how the modelling can contribute, simulation consideration is needed to evaluate in initial stage of semiconductor process. First principles model may be further classified into analytical models and numerical or computational models based on how the equations comprising the model are solved. The complexities in the geometries of the semiconductor process simulations are the key obstacles in modelling the fabrication processes of semiconductor devices like transistors, led, etc.which are the branches of automation design for electronics, and the part of its one of the sub-field is known as technology CAD (TCAD). Previous research is conduct by hardware experimental setup to develop the wafer crystal growth and the main gases use is $\mathrm{GaN}$. There is a considerable interest of Gallium Nitride for its broad band gapping and applications in the higher temperature electronics. From experimental hardware setups are successfully developed in the simulation setup for a reliable technology development recently. Researchers are focusing on the 
issues of improving the design geometry of the reactors and optimize the flow pattern uniformity into the reactors. Fluid flow models within both the horizontal as well as the vertical flow type MOVPE reactors have been published in several articles elaborately. Till now, only overviews of the previous processes which are MOVPE and HVPE growth reactors have been brought into forward. The HVPE method, defined as the potential growth technique so that both the large size as well as the thick crystal layers of GaN can be furnished (Zhao 2005). This is related to the fact that by this method, high growth rate is determined by mass input rate. It will cause melting of gallium through reaction of $\mathrm{GaN}$ at high temperature and near equilibrium process. While MOCVD process, produce lower growth rate with $\mathrm{NH}_{3}$ in precisely controlled ambient temperature and pressure. This method is non-equilibrium process and it is suitable for growth of device graded thin film. Instead of high expenses between MOCVD and HVPE processes in different chambers, it is possible to concurrently grow thick and thin base layers on target substrates and reduced surface contaminants. To address these issues, new development called MOHVPE has been proposed. These are the results of hybridization between the growth reactor for MOVPE and HVPE simultaneously.

\section{Growth chamber modeling}

Based on the findings from the literature review, the design considerations of the growth chamber can be divided into few stages. Before describing those stages, it is to be mentioned that the initial point is to define the assessment method, i.e. which method would be considered as suitable design for the growth chamber of MOHVPE process based on the requirements or purposes. Based on the previous designs of MOVPE and HVPE reactors' specifications are identified for the new modelling of MOHVPE reactor having horizontal duct. This modelling of the reactor was performed in a computer aided software tool named SOLIDWORKS (Version 2011). The design was based on two dimensional (2D) and three dimensional (3D) models where atmospheric pressure was assumed inside the reactor in order to overcome the computational time. This design consists of five nozzle inlet with each of its $6 \mathrm{~cm}$ long arranged in $40 \mathrm{~cm}$ length horizontal direction with one outlet tube where the gasses dispenser as seen in Fig. 1.

There are 3 substrate holders which are used to observe the uniformity and to investigate the effect of geometry during the flow as shown in Fig. 2. The nozzle inlets are divided by long beam plate to prevent the mixing of gases before entering the chamber area as it is presented in Fig. 2.

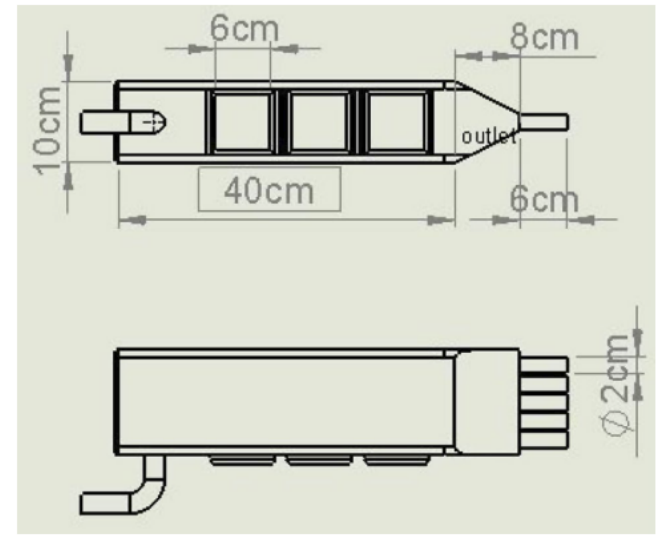

Fig. 1 Modelling dimension of metal organic halide vapor phase epitaxy (MOHVPE) reactor

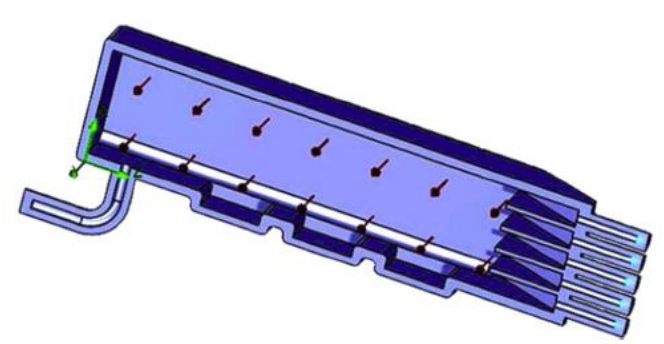

Fig. 2 Reactor modeling was performed based on 2D and 3D model with barrier between the inlet nozzles

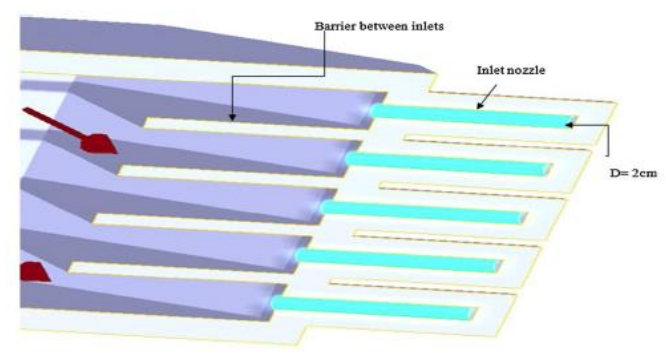

Fig. 3 Inlet nozzles as the main entrance

\section{Nozzle inlet modelling}

Nozzle inlet modeling in Fig. 3 is the major important part need to analyze for good expected as this chamber development with newly development as alternative for MOCVD 
and HVPE method. The main flow to consider is velocity and flow rate once pass by to enter the chamber and absorb wall will occur. Inlet velocity it is a common practice to design the inlet nozzle based on the momentum ( $\rho$ v2) of the entering fluid or air. The nozzle size depends on the presence of internals in the inlet nozzle. In every five single nozzle inlets inside the reactor, they are divided by long beam separation plates to prevent mixing of gases before entering the chamber area so they will meet shortly after the barrier passed by.

\section{Simulation of flow pattern in MOHVPE growth chamber}

The design of the chamber was used as the basis for FEM analysis, and its volume was divided into mesh elements in order to perform finite element analysis on its body. While creating the mesh of the chamber geometry, it is favourable to choose the mesh control, size of the element and their shape with global seed size is applied each part and element type is assigned. Mesh optimization process was performed to ensure the precision of the solver to obtain acceptable results. For an accurate result, a triangular mesh with a minimum element size $150 \mathrm{~nm}$ was used. Geometry of the chamber was solved using finite-element SolidWorks code 2011. Figure 4a, b shows the solution obtained using uniform meshing as obtained. Special attention has been appointed to capture the large fluctuations of the velocity in critical areas. But another type of mesh elements was obtained for the 3D geometries due to their design constraints. For this meshing representation, the studied region had more than 130,000.00(130K) elements.

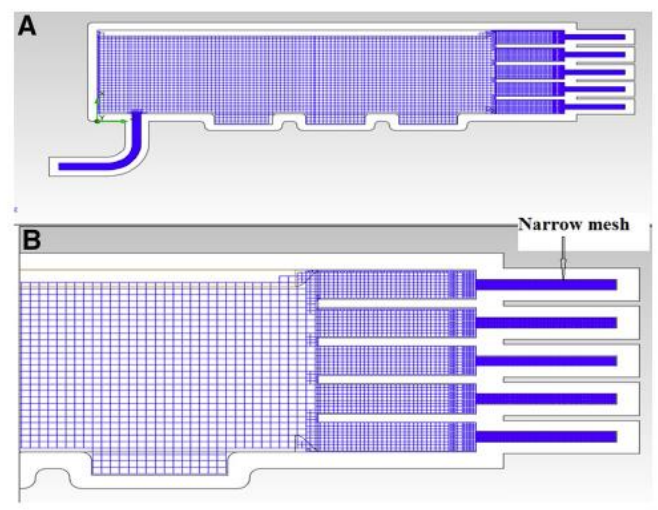

Fig. 4 Meshing generation geometry of Reactor chamber (a) Full body meshing (b) close up narrow inlet
The entire geometry of the reactor was not considered for the simulation owing to the rectangular symmetry; nevertheless, 2D simulation was not suitable for this case study. The inlets of $\mathrm{H}_{2}$ and $\mathrm{N}_{2}$ were dealt as the inflow boundary conditions presuming the flow of the gasses are laminar. $\mathrm{NH}_{3}$ also flows through inlet 4 and 5 ranking from the bottom chamber. Hydrogen is heated up to $800{ }^{\circ} \mathrm{C}$ before starting the operation of the real system as there is a requirement of cleaning the full chamber to prevent the parasitic deposition. The gas is presumed as Newtonian in the continuum regime. Thermocouple was utilized to monitor the temperature from the centre of the wafer holder to maintain the growth temperature at set point. When the gasses get into the reactor up to required volume level, they reach at the temperature level same as the wall temperature which represents the furnace heating conditions.

The inlet flow velocity is set as a constant quantity of $0.05 \mathrm{~m} / \mathrm{s}$ for all categories of simulations at each inlets. The temperature is varied between $1,273.2$ and $1,473.2 \mathrm{~K}$ in all the simulations run. In the meantime, the pressure is set as $0.1 \mathrm{MPa}$ for the opening of the pressure. Maintaining the heating system at every level is also necessitates due to risk of deposition of parasitic chlorides of ammonia. The chamber is divided into two zones with different

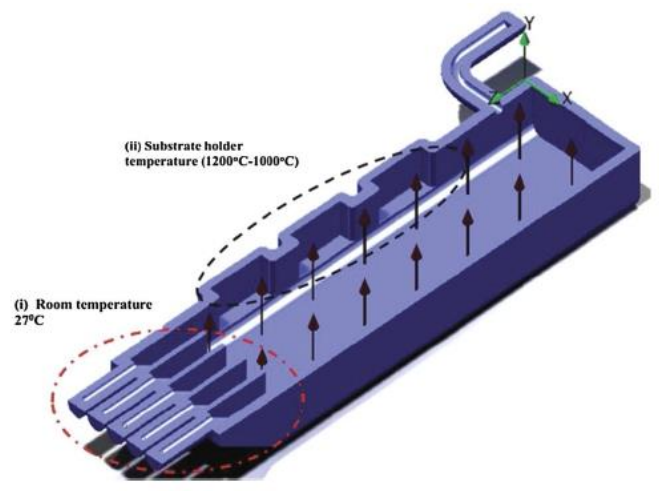

Fig. 5 Two zone area of temperature involved. (I) Inlet nozzle area (II) substrate holder area

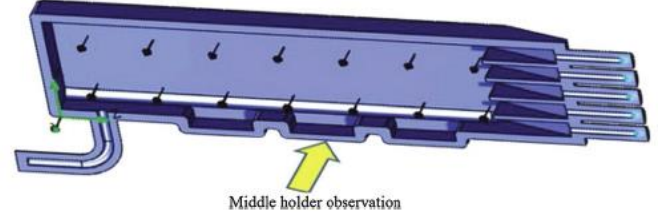

Fig. 6 Middle holder is observed in simulation run 
temperature. The inlets are extended towards the substrates as well as the temperature of the wall was set from $550{ }^{\circ} \mathrm{C}$; the 5 inlet nozzles are set for flow under the room temperature of $27{ }^{\circ} \mathrm{C}$ for each inlet. While after pass the barrier the temperature in heat up between 1,200 and $1,000{ }^{\circ} \mathrm{C}$ as seen in Fig. 5. Central holder is stressed out to make clear of the best position for the required activities as observed in Fig. 6. Because of the crucial radioactive heat generations from the hot substrates, it was indispensable to make use of the corresponding models for heat transfer phenomena.

\section{Analysis modeling}

The simulation results can be utilized to determine the minimized among the substrate holder locations and observed the flow pattern surrounding the reactor geometry model. The results obtained in the calculation include flow patterns, reaction rate inside the chamber and volumetric flow since it also depends on presence of adsorbed layer at the chamber itself.

As discussed before, this is a novelty development while the result and the parameter involved in the real life applications is focused only on velocity and temperature influenced flow pattern with pressure constant inside the chamber. For the first stage, results were obtained with $\mathrm{NH}_{3}$ and outlets respectively located at $24 \mathrm{~cm}(1), 34 \mathrm{~cm} \mathrm{(2)} \mathrm{and}$ $44 \mathrm{~cm}$ (3) from the inlet nozzle to substrate holder is seen in Fig. 7 with the temperature profile. The system operates in such that both processes do not happen simultaneously, but first the system needs to be purged with nitrogen is set temperature $800{ }^{\circ} \mathrm{C}$ by vacuuming the chamber for three times with both MOVPE and HVPE before the gases start to enter. This is to make sure that the whole chamber is completely clean and free from dust and other pollutants.

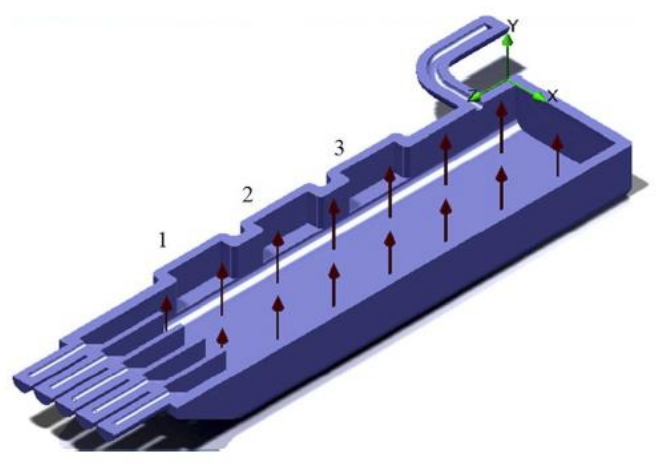

Fig. 7 2D cross section view with 3 substrate holder allocated

\section{Results}

The simulation results can be utilized to determine the minimized among the substrate holder locations and observed the flow pattern surrounding the reactor geometry model. The results obtained in the calculation include flow patterns, reaction rate inside the chamber and volumetric flow since it also depends on presence of adsorbed layer at the chamber itself.

As discussed before, this is a novelty development while the result and the parameter involved in the real life applications is focused only on velocity and temperature influenced flow pattern with pressure constant inside the chamber. For the first stage, results were obtained with $\mathrm{NH}_{3}$ and outlets respectively located at $24 \mathrm{~cm}(1), 34 \mathrm{~cm} \mathrm{(2)} \mathrm{and}$ $44 \mathrm{~cm}$ (3) from the inlet nozzle to substrate holder is seen in Fig. 7 with the temperature profile. The system operates in such that both processes do not happen simultaneously, but first the system needs to be purged with nitrogen is set temperature $800{ }^{\circ} \mathrm{C}$ by vacuuming the chamber for three times with both MOVPE and HVPE before the gases start to enter. This is to make sure that the whole chamber is completely clean and free from dust and other pollutants.

Thus, the zone temperature was assigned after cleaning the inlet tubes preventing from getting muggy and tends to allow the gases to stick over the walls of the growth chamber. Flow through inlet duct typically is stable at the entrance of chamber but then the growth becomes rapidly faster after passing the inlet.

\section{Geometry factor}

Thermal decomposition due to reactions take place in reaction chambers thus generation of thin films experienced as desired output. The geometry of the reactor has significant influence on the flowing fluid's flow characteristics. Therefore, it is essentially regarded to optimize the reactor's shape to ensure smooth as well as laminar flow inside the chamber. The MOHVPE processes of the reactor posses several parameters that necessitate the careful design as well as optimization of the processes' parameters for effectively acceptable operation. Depending on the geometric factors for modelling, principal parameters which involve are the inlet area, the distance between the nozzles and the position of substrates' holders etc. critically prevent the recirculation in flow pattern. These critical parts are shown in the Fig. 8.

\section{Temperature effect surrounding the chamber}

The temperature of the surrounding chamber is influenced by temperature of the chamber's body. The reactor is set to 
Fig. 8 Part of the critical that possibly affect the geometry

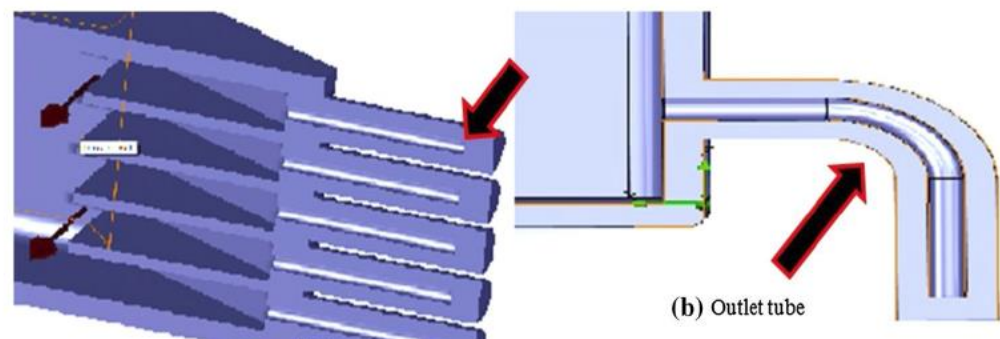

(a) Inlet nozzle

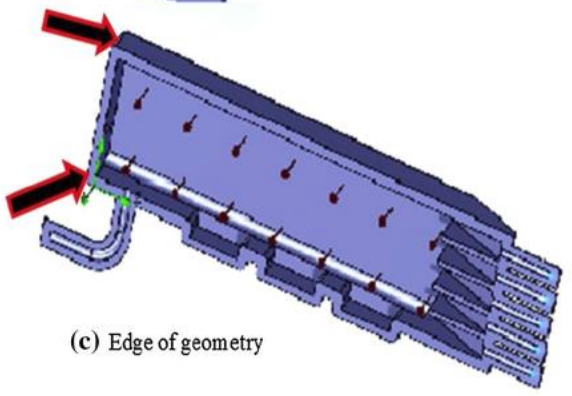

Fig. 9 Final temperature distribution at $1,473 \mathrm{~K}$

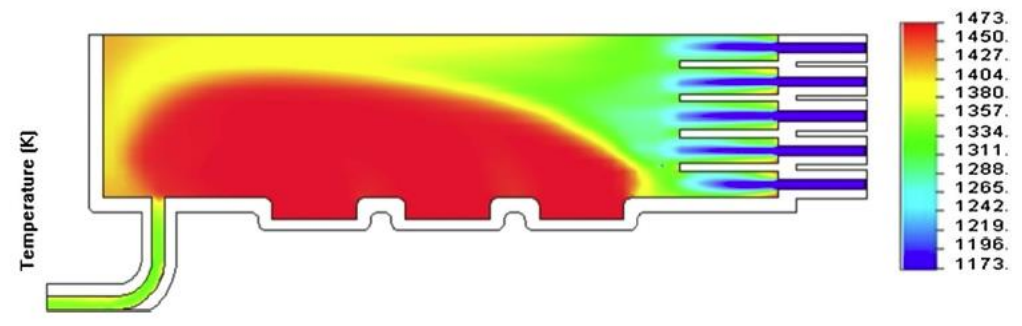

control the temperature in a constant profile through reflex responses or changes (either raise or lower) as required. The main part of the geometrical effects with simulation analysis is based on up and down temperature rate surrounding it and would lead towards poor retention. Based on this phenomenon, the observation on temperature rate is analysed from 1,200 to $1,000{ }^{\circ} \mathrm{C}$ and it explained the three situations of temperature.

\section{Simulation result at $1,473 \mathrm{~K}$}

The simulation results with the inlet fluid temperature of $1,473 \mathrm{~K}\left(1,200{ }^{\circ} \mathrm{C}\right)$ could be observed in the Fig. 9. Here, the colour contour is showing the simulation model's temperature fluctuation at that given condition. The low temperature is indicated by the blue color then it will turned into light blue, green, yellow and at the end it is red color indicating the high temperature band/contour in the regions. The simulation result is indicating the 5 inlet flows of fluid and air is under controlled temperature since it is set to be under room temperature. After a few iterations of the reaction chamber's interactive computation, the temperature appears to be rising through the lengthwise direction of the reaction chamber. After certain period of time the flow pattern grown up slowly to red color surrounding the chamber, It can be seen in the result where the contour of the color starts changing from red to yellow, green and last one is light green before it flows out of the tube outlets under the chamber itself to purge out all the unnecessary fluid at the end of the reaction chamber.

The result shows that the distribution of the temperature in reaction chamber seems decreasing at constant gradient. It could be visually perceived that the temperature distribution on substrate is not uniform and the temperature increase and turn to red chilis around the substrate after

\section{Link to Full-Text Articles:}

http://link.springer.com/article/10.1007/s00542-013-2046-y 